\title{
UHECR from high- and low-luminosity Gamma-Ray Bursts
}

\section{Annika Rudolph, ${ }^{a, *}$ Jonas Heinze, ${ }^{a}$ Daniel Biehl, ${ }^{a}$ Anatoli Fedynitch, ${ }^{a, b}$ Denise

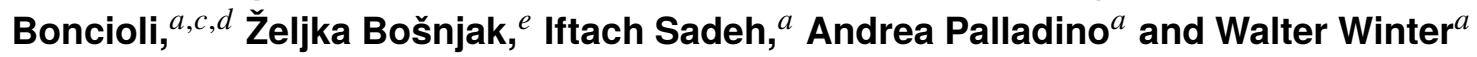

${ }^{a}$ Deutsches Elektronen-Synchrotron DESY,

Platanenallee 6, 15738 Zeuthen, Germany

${ }^{b}$ the University of Tokyo, Institute for Cosmic Ray Research

5-1-5 Kashiwa-no-ha, Kashiwa, Chiba 277-8582, Japan

${ }^{c}$ Università degli Studi dell'Aquila, Dipartimento di Scienze Fisiche e Chimiche, via Vetoio, L’Aquila, Italy

${ }^{d}$ INFN Laboratori Nazionali del Gran Sasso, Assergi (L'Aquila), Italy

${ }^{e}$ University of Zagreb, Faculty of Electrical Engineering and Computing, Unska ul. 3, 10000 Zagreb, Croatia

E-mail: annika.rudolph@desy.de

For a long time Gamma-Ray Bursts (GRBs) have been discussed as potential origin of UltraHigh-Energy Cosmic Rays (UHECRs). This paradigm is challenged by the lack of High-Energy (HE) neutrinos detected by IceCube that could be associated with known GRBs. In this work, we study two scenarios that could reconcile GRBs as UHECR sources: First we fit the UHECR spectrum and $\left\langle X_{\max }\right\rangle$ as observed by Auger with conventional long-duration GRBs in a multi-zone internal shock model. Here, we systematically scan over different engine realisations that may be distinguished by their light curves. We show that a good fit is possible for a broad range of parameters if $\sigma\left(X_{\max }\right)$ is not included and that the corresponding neutrino fluxes are in reach of future instruments such as IceCube Gen2. In this context, we critically review the required engine kinetic energy. Second, we explore the prompt phase of Low-Luminosity GRBs (LL-GRBs) as potential sources of Very-High-Energy (VHE) gamma-rays and UHECR. For this purpose, we model a LL-GRB with properties similar to GRB 980425 within the internal shock model and vary the fraction of internal energy supplying the magnetic field. We find that for strong magnetic fields, iron nuclei (protons) can be accelerated up to $10^{11} \mathrm{GeV}\left(10^{9} \mathrm{GeV}\right)$, while for low magnetic fields a VHE component potentially observable by Imaging Air Cherenkov Telescopes (IACTs) could be produced.

$37^{\text {th }}$ International Cosmic Ray Conference (ICRC 2021)

July 12 th - 23rd, 2021

Online - Berlin, Germany

\footnotetext{
*Presenter
} 


\section{Introduction}

To accelerate cosmic rays via shocks to the highest energies of $>10^{18} \mathrm{eV}$, the size of a potential source needs to be smaller than the particle gyroradius [1]. By this requirement alone many astrophysical objects are ruled out as Ultra-High-Energy Cosmic-Ray (UHECR) sources. Among the remaining are Gamma-Ray Bursts (GRBs), jetted outbursts with $E_{\text {iso }}$ between $10^{50}$ to $10^{55} \mathrm{erg}$ in gamma-rays and durations between fractions of a second to a few hundred seconds. Their potential for acceleration of the highest-energy particles has been discussed for a long time, see for example [2]. In a multi-messenger context, the UHECR-GRB paradigm is challenged by the non-association of High-Energy (HE) neutrinos detected by IceCube with known GRBs [3, 4]. The neutrino stacking limit can be translated in a neutrino production limit that can be satisfied either by a low baryon contamination of the outflow or by a low neutrino production efficiency.

In simple one-zone models, this translates into strong constraints on the energy transferred to non-thermal baryons, the energy dissipation radius or Lorentz factor of the emitting plasma. For example [5] have shown that a successful fit to the UHECR energy spectrum by GRB sources is possible for low isotropic gamma-ray luminosities or large emission radii. Several publications discuss Low-Luminosity GRBs (LL-GRBs) with isotropic luminosities of $L_{\text {iso }}<10^{49} \mathrm{erg}$ as a distinct source class which can power the observed UHECR and/or neutrino flux [6-10]. However, $[11,12]$ argue that cosmic rays in these objects may not reach ultra-high energies . Multi-zone models may alleviate the tension between neutrino bounds and cosmic-ray fits by decoupling the production radii of the different particle species; see for example [13, 14]. A successful fit to the UHECR spectrum and mass composition has been performed in [15].

In the following we explore two scenarios that reconcile GRBs as acceleration sites of UHECR: first, we introduce results based on [16], where we study conventional long GRBs in a multi-zone model. Systematically scanning over a parameter space of engine realisations, we calculate the injection fractions of different mass groups necessary to fit the observed UHECR spectrum; and second, considering the prompt phase of LL-GRBs as potential target for multi-wavelength and multi-messenger astronomy (results based on [17]). For this purpose, we model a prototype to reproduce observations of a real GRB, imposing different magnetic field strengths via the microphysics parameter $\epsilon_{B}$. We calculate the observed spectra and light curves with a focus on a Very-High-Energy (VHE) component potentially observable by Imaging Air Cherenkov Telescopes (IACTs). The results of the (leptonic) radiation modeling are then used to determine the maximal energies achievable for different cosmic-ray nuclei.

\section{Fitting the UHECR spectrum with different engine realisations in a multi-zone model}

In the present framework, GRBs are modeled within the internal shock model $[18,19]$, whereas the outflow is discretized as a series of plasma shells. The observed radiation is produced in collisions of plasma shells due to their different velocities. The initial Lorentz factor distribution as a function of radius (or distance to the central emitter) may be viewed as an imprint of the engine activity and directly reflects in the observed light curve. We assume a prototype of fixed duration and luminosity to study the impact of the engine realisations. The initial Lorentz factor 

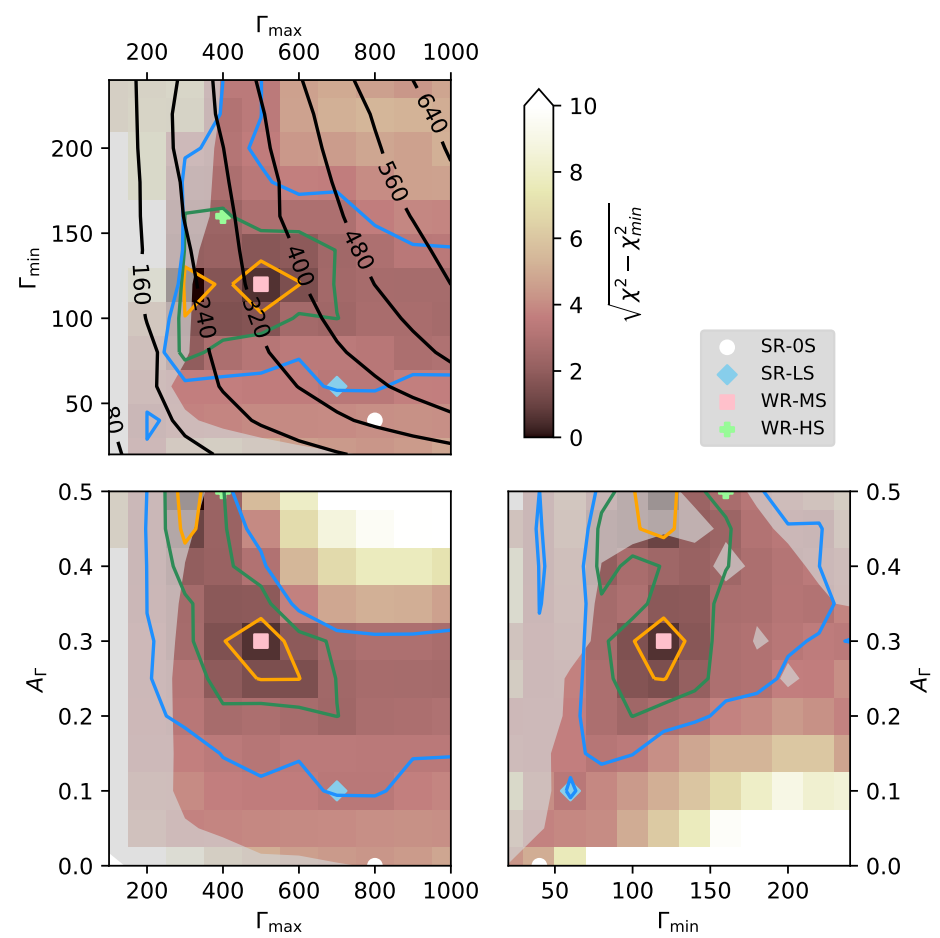

Figure 1: Left: Illustration of the initial Lorentz factor distribution and parameters of the scan: $\Gamma_{\min }, \Gamma_{\max }$ and $A_{\Gamma}$. Right: Result of the parameter space scan and fit to the UHECR spectrum and $\left\langle X_{\max }\right\rangle$. The black lines in the upper plot show the bulk Lorentz factor of the outflow, the coloured lines the 1 to $3 \sigma$ contours (blue $-3 \sigma$, green $-2 \sigma$, orange $-1 \sigma$ ). The points (with legend on the upper right) are representative examples used to illustrate the results for prototype engines. In the grey shaded regions, more than $40 \%$ of the energy is dissipated in the optically thick regime. Figure taken from [16].

distribution is varied using three parameters: the amplitude of stochastic oscillations $\left(A_{\Gamma}\right)$, and the maximal and minimal Lorentz factors $\Gamma_{\min }$ and $\Gamma_{\max }$. This is illustrated in Fig. 1 (a). Scanning over the $A_{\Gamma}, \Gamma_{\min }$ and $\Gamma_{\max }$ parameter space, we perform a fit to the UHECR spectrum and the first moment of the distributions of the maximum of the shower depth $\left\langle X_{\max }\right\rangle$ as observed by the Pierre Auger Observatory [20], assuming a GRB distribution following [21], with a similar luminosity of $\approx 10^{52.5} \mathrm{erg} / \mathrm{s}$ for each GRB. Free fit parameters are the initial (at the base of the outflow) injection luminosities of different elements $(\mathrm{H}, \mathrm{He}, \mathrm{O}, \mathrm{N}, \mathrm{Si}$ and $\mathrm{F})$ and the baryonic loading (the fraction of the generated internal energy supplying non-thermal cosmic rays divided by the fraction of energy going into photons). Since the conversion efficiency between the initial kinetic energy of the outflow to the observed gamma-ray radiation depends on the initial properties of the outflow, the initial jet kinetic energy is adjusted to keep the overall energy transferred to photons $E_{\gamma \text {,tot }}$ constant. Motivated by observations, the photon field is assumed as a broken power law of comoving peak energy $1 \mathrm{keV}$ and spectral indices $\alpha=-1$ and $\beta=-2$. Cosmic rays injected as a power law of index $p=-2$. The maximal energy is determined balancing energy losses and acceleration (in the Bohm limit, thus $t_{\mathrm{acc}}^{\prime}=\frac{\gamma^{\prime} m c}{e B^{\prime}}$ where primed quantities are in the plasma comoving frame). The model accounts for photo-disintegration, photo-pair and photo-pion production, synchrotron and adiabatic 

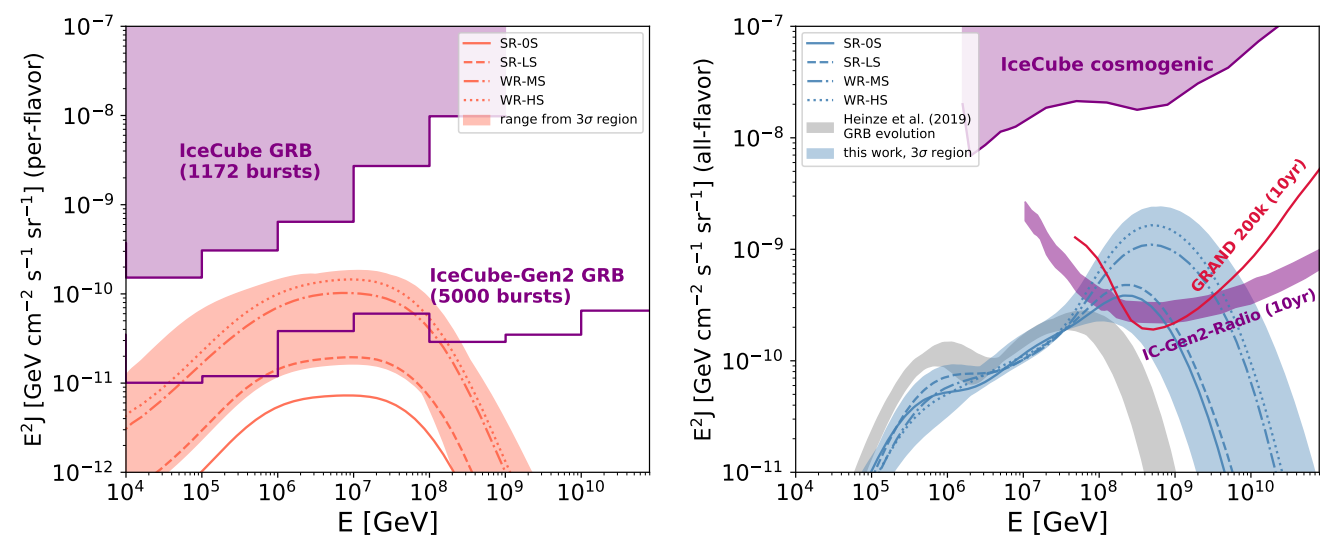

Figure 2: Single-flavour prompt (left) and the all-flavour cosmogenic (right) neutrino fluxes for the 3- $\sigma$ CL contour from Fig. 1. In the left Figure we show the current IceCube stacking limit [4] and the projected IceCube Gen2 limit for 5000 bursts [24]. In the right Figure, the current IceCube cosmogenic limit is taken from [24], the future limits from [24, 25]. For comparison we include the expected neutrino flux for a rigitidy-dependent UHECR fit as in [22]. Figure taken from [16]

losses. The interactions in the source are iteratively computed with the NeuCosmA code [5]. The propagation between source and Earth is calculated with PRINCE [22], the interaction model in both codes is constructed from TALYS [23].

As can be inferred from Fig. 1, we find a good fit to the UHECR spectrum and composition for a broad range of parameters. Overall, a bulk Lorentz factor between 200 and 400 and moderate stochasticity are favoured. The required heavy mass fraction (of nuclei heavier than Helium) at injection is found to be $70 \%(95 \% \mathrm{CL})$ and a typical baryonic loading of order $f_{p}=10-100$. A high level of stochasticity translates into large contrasts of initial Lorentz factors between neighbouring shells. This induces collisions close to the central emitter with high particle densities that result in efficient photo-disintegration. Hence engines with high (low) stochasticity require a high injection luminosity of heavy (light) nuclei to fit the observed UHECR composition. The observations for $\left\langle X_{\max }\right\rangle$, which indicate that the composition gets heavier with increasing energy, are well reproduced in our models. However, $\sigma\left(X_{\max }\right)$ data implies that the change in composition has to be gradual, with little overlap of neighbouring mass groups. This cannot be achieved in our model, mainly due to two reasons. First, the overall emitted spectrum is a sum of all spectra emitted in single collisions. Thus many overlapping spectra are summed to one, which effectively creates a soft spectrum. Second, especially for stochastic engines with a high level of disintegration, different mass groups overlap in single collisions. We point out that similar effects would be expected if different source classes or strong differences among the properties of GRBs were considered.

By calculating the in-source and cosmogenic neutrino fluxes self-consistently, we find no tension with current neutrino limits, see Fig. 2. The predicted fluxes are within the sensitivity of upcoming telescopes such as IceCube Gen2 or GRAND. For stochastic engines, the same highdensity collisions that induce a high level of photo-disintegration also result in efficient neutrino production. Thus, the predicted neutrino fluxes are higher in these cases. The stochasticity will also be reflected in the observed gamma-ray light curves which may thus be used to discriminate 

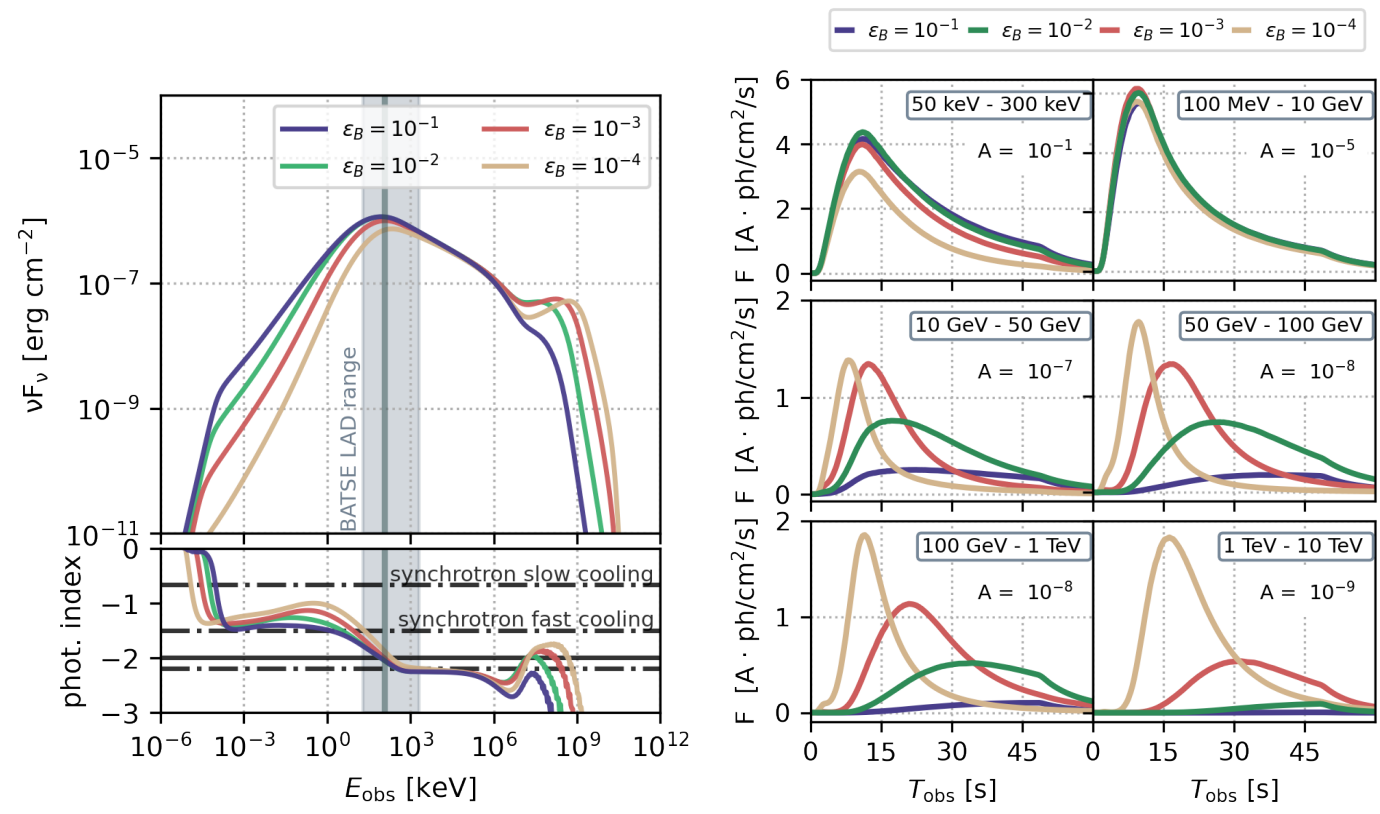

Figure 3: Prototype with observed properties similar to GRB 980425. Left: Simulated spectrum $v F_{v} \propto$ $E^{2} d N / d E$ and photon index (for a power law of $d N / d E$ ). We indicate the observed peak energy of the reference event with a vertical line, the energy range of the observing instrument is shown as a grey shaded region. Right: Light curve in the sub-MeV band and for different high-energy to very-high-energy bands. In both figures we show the results for different values of the microphysics parameter $\epsilon_{B}$, while the dynamical evolution of the outflow was kept constant. Figure taken from [17]

between engine realisations.

If GRBs are the dominant sources of the UHECR flux, a local emissivity of $\sim 10^{44} \mathrm{erg} \mathrm{Mpc}^{-3} \mathrm{yr}^{-1}$ for a local GRB rate of $\sim 1 \mathrm{Gpc}^{-3} \mathrm{yr}^{-1}$ is required. In this case each GRB has to emit $2-3 \cdot 10^{53} \mathrm{erg}$ in UHECR baryons. Taking into account the efficiency in our model, which ranges between $\sim 10 \%$ to $30 \%$, the initial kinetic energies of the outflow have to be of order $10^{55}-10^{56} \mathrm{erg}$. While this is a very aggressive assumption, it is unavoidable if GRBs are the sources of UHECR. A cosmic-ray injection spectrum harder than $p=-2$ would reduce the required kinetic energy.

\section{Low-luminosity GRBs in a multi-messenger context}

To explore LL-GRBs as potential targets for multi-messenger and multi-wavelength astronomy, we simulated three prototypes with observed properties similar to the observed GRBs GRB 980425, GRB 100316D and GRB 120714A within the internal shock model. Here, we show only the results for the prototype based on GRB 980425 while referring to our full publication for the other GRBs [17].

Our simulated GRB has a single-peaked light curve of approximately same $T_{90}$ as GRB 980425, also the peak energy of $E_{\text {peak }}=122 \mathrm{keV}$ and the observed fluence are similar to the reference event. We expect a strong impact of the magnetic field $B^{\prime}$ on a potential VHE component for fixed properties of the dynamical outflow (defining the Lorentz factor, comoving density $\rho^{\prime}$ and energy density $\varepsilon_{\text {diss }}^{\prime}$ 
at a given distance from the central emitter and time, all calculated following [18]). To investigate this behaviour, we systematically vary the fraction of energy supplying the magnetic field, set by the microphysics parameter $\epsilon_{B}$ while keeping the observed quantities ( $E_{\text {peak }}$ and fluence) approximately constant. From $\epsilon_{B}$ the magnetic field in a dissipation region can be calculated as $B^{\prime}=\sqrt{8 \pi \varepsilon_{\text {diss }}^{\prime} \epsilon_{B}}$. The radiative calculations are performed with the time-dependent AM3-Code [26] which includes synchrotron and inverse Compton radiation, as well as photon-photon pair annihilation (with a full feedback of the secondary lepton pairs) and adiabatic cooling. Absorption on the Extragalactic Background Light (EBL) is calculated with the open-source GAMMAPY package [27, 28].

The predicted spectrum and light curve in diferent energy bands from gamma-ray to VHE gamma-ray are shown in Fig. 3. We find that for otherwise fixed jet properties, large values of $\epsilon_{B}$ (translating into large magnetic fields) result in a relatively weak VHE component. On the other hand, for low $\epsilon_{B}$ we predict VHE fluxes that despite overall low luminosity are within the sensitivity of instruments such as CTA [29]. We point out that if we require efficient synchrotron radiation of electrons (i.e. to be in the fast-cooling regime) this puts lower bounds on $\epsilon_{B}$. Additional probes of the magnetic field could be (1) the spectral index $\alpha$ below the $\mathrm{MeV}$ peak (the spectrum gets steeper with increasing $\epsilon_{B}$, lower panel of Fig. 3 (a)), (2) the flux in the optical and UV (which increases with increasing $\epsilon_{B}$, upper panel of Fig. 3 (a)) or (3) a delayed onset of the (very)-high-energy components (for increasing $\epsilon_{B}$, the early suppression of the VHE component gets stronger, see Fig. 3 (b)).

Using the results from the leptonic radiation modeling and the same dynamical evolution of the fireball, we calculate the maximal energies achievable for cosmic-ray nuclei (in the source frame), again for different values of $\epsilon_{B}$. The result (as a function of the distance to the central emitter) is shown in Fig. 4, where each collision is represented by one dot. For $\epsilon_{B}=0.1$ protons can be accelerated up to energies of $\approx 10^{9} \mathrm{GeV}$, iron up to $\approx 10^{11} \mathrm{GeV}$. The highest energies are reached at large distances from the source. We point out that the observed $\gamma$-rays are produced at intermediate radii around $10^{14} \mathrm{~cm}$, where cosmic-ray energies are significantly smaller. Simplified one-zone models (in which the photon spectrum and the cosmic-ray spectrum are produced are at the same radius) will thus under-estimate the true maximal cosmic-ray energies. On the other hand, as most of the energy is dissipated at lower radii, the bulk of cosmic rays will not achieve the highest energies.

\section{Conclusion}

In this work, we have explored GRBs as potential sources of UHECR. Conventional long high-luminosity GRBs were explored in a multi-zone model following [16]. Successful fits to the UHECR spectrum and $\left\langle X_{\max }\right\rangle$ were demostrated for a broad range of engine realisations which differ in the initial Lorentz factor distribution of the outflow. The required heavy mass fraction injected at the base of the jet is above $70 \%(95 \% \mathrm{CL})$, where engine realisations/ light curves with high stochasticity induce more photo-disintegration, thus require a higher injection of heavy nuclei. The corresponding prompt and cosmogenic neutrino fluxes expected at Earth are below the current neutrino stacking limits but within the sensitivity of upcoming telescopes such as Ice-Cube Gen2.

Our second focus were LL-GRBs. Incorporating a fully self-consistent leptonic model of the observed sub-MeV photon spectra in a multi-zone model, we have shown that protons (iron nuclei) 

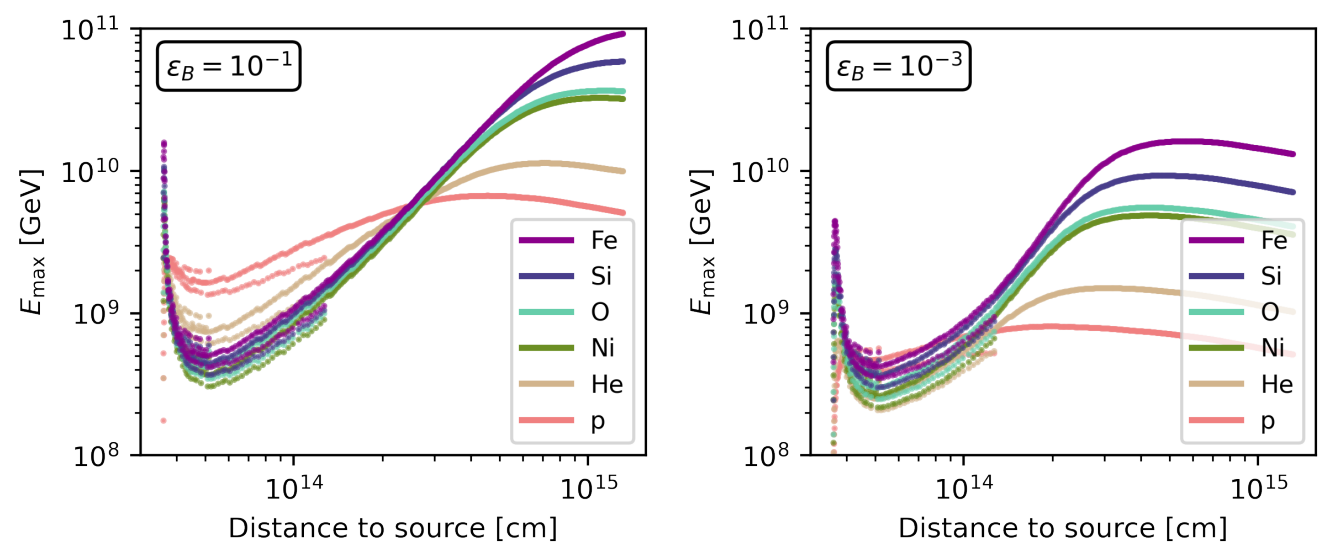

Figure 4: Maximum energies of cosmic-ray nuclei in the source frame for the simulated prototype based on GRB 980425 for a fixed jet dynamical evolution, varying the fraction of the energy supplying the magnetic field (set by $\epsilon_{B}$ ). Figure taken from [17]

can reach energies up to $10^{9}\left(10^{11}\right) \mathrm{GeV}$. Varying the fraction of the internal energy supplying the magnetic field, we have made the connection between the maximal cosmic-ray energies and multiwavelength predictions, with a special focus on a Very-High-Energy (VHE) component potentially observable by ground-based instruments. Despite their low peak energies and luminosities, we have found a high-energy component potentially observable by current and future instruments. This component is strong for weak magnetic fields (for which lower maximal cosmic-ray energies were found). Thus, in our model a LL-GRB can either accelerate cosmic rays to the highest energies or produce a strong VHE component. Additionally we have pointed out how the optical flux and the spectral shape below the sub-MeV peak can be used as complementary probes of the magnetic field strength.

We point out that our results do not take into account hadronic feedback on the observed SED. However, for example [30] have shown that the baryonic loading cannot be too high, as otherwise the spectral shape is incompatible with observations. While this was beyond the scope of this study, it may be addressed in a future publication.

\section{References}

[1] A.M. Hillas, Ann. Rev. Astron. Astrophys. 22 (1984) 425.

[2] E. Waxman, Phys. Rev. Lett. 75 (1995) 386.

[3] ICECube collaboration, Astrophys. J. 805 (2015) L5.

[4] IceCube collaboration, Astrophys. J. 843 (2017) 112.

[5] D. Biehl, D. Boncioli et al., Astron. Astrophys. 611 (2018) A101.

[6] K. Murase, K. Ioka et al., Astrophys. J. 651 (2006) L5.

[7] R.-Y. Liu, X.-Y. Wang et al., Mon. Not. Roy. Astron. Soc. 418 (2011) 1382.

[8] N. Senno, K. Murase et al., Phys. Rev. D93 (2016) 083003. 
[9] B.T. Zhang, K. Murase et al., Phys. Rev. D97 (2018) 083010.

[10] D. Boncioli, D. Biehl et al., Astrophys. J. 872 (2019) 110.

[11] F. Samuelsson, D. Bégué et al., Astrophys. J. 876 (2019) 93.

[12] F. Samuelsson, D. Bégué et al., Astrophys. J. 902 (2020) 148.

[13] M. Bustamante, K. Murase et al., Astrophys. J. 837 (2017) 33.

[14] A. Rudolph, J. Heinze et al., Astrophys. J. 893 (2020) 72.

[15] N. Globus, D. Allard et al., Mon. Not. Roy. Astron. Soc. 451 (2015) 751.

[16] J. Heinze, D. Biehl et al., Mon. Not. Roy. Astron. Soc. 498 (2020) 5990.

[17] A. Rudolph, Ž. Bošnjak et al., arXiv e-prints (2021) arXiv:2107.04612.

[18] F. Daigne and R. Mochkovitch, Mon. Not. Roy. Astron. Soc. 296 (1998) 275.

[19] S. Kobayashi, T. Piran et al., Astrophys. J. 490 (1997) 92.

[20] The Pierre Auger Observatory: Contributions to the 35th International Cosmic Ray Conference (ICRC 2017), 8, 2017.

[21] D. Wanderman and T. Piran, Mon. Not. Roy. Astron. Soc. 406 (2010) 1944.

[22] J. Heinze, A. Fedynitch et al., Astrophys. J. 873 (2019) 88.

[23] "TALYS-1.0", Proceedings of the International Conference on Nuclear Data for Science and Technology - ND2007, EDP Sciences (2008) 211-214. 10.1051/ndata:07767.

[24] ICeCube-Gen2 collaboration, J. Phys. G 48 (2021) 060501.

[25] GRAND collaboration, Sci. China Phys. Mech. Astron. 63 (2020) 219501.

[26] S. Gao, M. Pohl et al., Astrophys. J. 843 (2017) 109.

[27] CTA Consortium collaboration, PoS ICRC2017 (2018) 766.

[28] C. Nigro et al., Astron. Astrophys. 625 (2019) A10.

[29] CTA Consortium collaboration, PoS ICRC2019 (2021) 673.

[30] K. Asano, S. Inoue et al., Astrophys. J. 699 (2009) 953. 